\title{
Utilité d'une typologie des exploitants artisanaux de bois pour contribuer à la formulation d'une politique publique en province Orientale (RD Congo)
}

\author{
Pitchou Tshimpanga Ongona ${ }^{(1)}$, Guillaume Lescuyer ${ }^{(2,3)}$, Jason Vleminckx ${ }^{(4)}$, \\ Bernard Adebu Abdala ${ }^{(5)}$, Dimandja Lokombe $\dagger^{(6)}$ \\ (1) Université de Kisangani (UNIKIS). Faculté de Gestion des Ressources Naturelles Renouvelables (FGRNR). Avenue \\ Kitima, 3. Kisangani (RDC).E-mail : pitchoutshim@gmail.com \\ (2) Centre de coopération Internationale en Recherche Agronomique pour le Développement (CIRAD). UR B\&SEF. BP 2527. \\ Yaoundé (Cameroun). \\ ${ }^{(3)}$ Center for International Forestry Research (CIFOR). P.O. Box 2008. Yaoundé (Cameroun). \\ (4) Université Libre de Bruxelles (ULB). Laboratoire d'Évolution biologique et Écologie. Avenue Franklin Roosevelt, 50. \\ BE-1050 Bruxelles (Belgique). \\ (5) Organisation Concertée des Écologistes et Amis de la Nature (OCEAN). Avenue de la Reine, 10. Kisangani (RDC). \\ ${ }^{(6)}$ ISEA-Bengamisa. Boulevard du 30 juin, 53. Kisangani, province de la Tshopo (RDC).
}

Reçu le 12 novembre 2015, accepté le 23 aout 2016.

Description du sujet. Cet article traite de la typologie des exploitants artisanaux de bois en vue d'aider à la formulation d'une politique publique en province Orientale (RDC).

Objectifs. L'objectif est de caractériser l'exploitation artisanale de bois d'œuvre pour proposer la mise en place de voies légales afin d'accroitre sa contribution à l'économie nationale.

Méthode. Des enquêtes ont été réalisées avec 412 exploitants artisanaux actifs dans 23 villages de la province Orientale entre 2010 et 2012. Une analyse factorielle des données mixtes (FAMD) et la classification hiérarchique des composantes principales (HCPC) ont été réalisées.

Résultats. Trois types d'exploitants artisanaux sont distingués : les scieurs d'origine provinciale ravitaillant les marchés de Kinshasa, les scieurs d'origine locale desservant les marchés locaux et les scieurs originaires des autres provinces que la province Orientale approvisionnant les marchés du Kivu et d'Ouganda.

Conclusions. La prise en compte de la spécificité de chacun de ces groupes d'opérateurs artisanaux permet d'améliorer l'élaboration et la mise en œuvre des mesures de politique publique nécessaires pour sécuriser et formaliser l'activité.

Mots-clés. Politique économique, marché des produits de base, récolte du bois, gestion des ressources naturelles, République Démocratique du Congo.

Utility of a typology of timber artisanal loggers, in order to contribute to the formulation of public policy on logging in Orientale province (DRC)

Description of the subject. This paper discusses the types of wood artisanal loggers, in order to assist in the formulation of public policy on logging in Orientale province (DRC).

Objectives. The objective was to characterize artisanal timber in order to propose the establishment of legal channels to increase the contribution of this product to the national economy.

Method. Surveys were conducted with 412 artisanal loggers active in 23 villages in Orientale province between 2010 and 2012. Factor analyses for mixed data (FAMD) and hierarchical classification on principal components (HCPC) were performed.

Results. Three types of artisanal loggers were distinguished: (1) sawyers of provincial origin who supply local markets in Kinshasa; (2) sawyers of local origin that serve local markets; (3) sawyers from the other provinces of Orientale province, supplying markets in Kivu and Uganda.

Conclusions. Taking into account the specificity of each of these groups of artisanal operators could improve the development and implementation of public policy measures needed to secure and formalize logging activity.

Keywords. Economic policies, commodity markets, logging, natural resources management, Democratic Republic of Congo. 


\section{INTRODUCTION}

En République démocratique du Congo (RDC), comme dans toute l'Afrique centrale, la gestion durable des ressources forestières s'est principalement traduite par l'aménagement des forêts de production concédées à des opérateurs industriels (Debroux et al., 2007 ; Van Acker, 2013). Cette production est principalement destinée à l'exportation vers l'Europe et l'Asie. Il existe cependant en parallèle un secteur domestique de grande ampleur qui relève largement de pratiques artisanales informelles. En RDC, le sciage artisanal est douze fois plus important en volume que la production industrielle enregistrée par l'administration (Lescuyer et al., 2014). Et pourtant, ce secteur ne fait pas l'objet de politiques publiques spécifiques.

Plusieurs raisons sont invoquées pour expliquer l'existence et l'extension du sciage artisanal informel en RDC :

- les différents conflits armés qu'a connus le pays durant les deux dernières décennies ont favorisé le développement des activités non régulées (Debroux et al., 2007 ; Nkoy, 2007) ;

- la croissance démographique et l'urbanisation entretenant une demande croissante pour des sciages bon marché, notamment dans les villes (Bayol et al., 2012) ;

- une règlementation imprécise, incomplète et largement inappliquée suite à l'absence de contrôles et au manque de connaissance des différentes parties prenantes (Djiré, 2003 ; Lescuyer et al., 2014).

La formalisation du secteur de sciage artisanal est généralement recommandée pour sécuriser l'exploitation artisanale, accroître sa contribution à l'économie nationale et contrôler ses impacts environnementaux (Benneker et al., 2012; Tiayon et al., 2012). La littérature tend toutefois à considérer le sciage artisanal comme une activité homogène. À titre d'exemple, Global Witness (2012), dans des enquêtes réalisées dans la province du Bandundu, assimile le sciage artisanal à l'utilisation illicite par des sociétés industrielles de permis d'exploitation artisanale. Par contre, certains chercheurs, à l'instar de Nkoy (2007) ou Belesi et al. (2013), considèrent que l'exploitation artisanale n'est pratiquée que par des scieurs dépourvus de permis et de façon relativement anarchique. Ces visions monolithiques du sciage artisanal influenceraient l'élaboration et la mise en œuvre des mesures de politique publique visant à réguler et à valoriser le secteur. Ce dernier recouvre des dynamiques différentes - voire opposées - que cette étude tente de présenter dans le cadre de la province Orientale, haut lieu du sciage artisanal en RDC (Benneker et al., 2012 ; Lescuyer et al., 2014).
Après la description de méthodes d'enquête et d'analyse, une typologie des scieurs artisanaux est dégagée à travers un jeu de variables descriptives. Cette discrimination des scieurs est utilisée pour identifier des approches et des instruments spécifiques en vue de sécuriser, pérenniser et formaliser le secteur du sciage artisanal.

\section{MÉTHODE}

\subsection{Site d'étude et collecte des données}

Deux méthodes d'enquête ont été employées pour la collecte des données. Dans un premier temps, des entretiens semi-dirigés individuels et anonymes ont été réalisés avec 412 exploitants artisanaux actifs dans 23 villages de la province Orientale ${ }^{1}$ (Figure 1) entre 2010 et 2012 . Étant donné l'absence de base de sondage pour les exploitants artisanaux, un échantillonnage en grappe a été préconisé à partir des 23 villages recensés. À l'issue de premières enquêtes organisées dans les sites d'exploitation artisanale de bois, localisés non loin de Kisangani, la liste des villages a été dressée. Tous les exploitants disponibles rencontrés dans ces villages ont été retenus pour les enquêtes. Les entretiens ont permis de caractériser le contexte socio-économique de chacun des exploitants.

En deuxième lieu, les 412 exploitants ont fourni des renseignements relatifs aux avantages et couts économiques de 439 opérations d'exploitation artisanale effectuées durant la période de la recherche.

\subsection{Analyse des données}

Les données obtenues ont été saisies avec le logiciel Excel puis analysées avec le logiciel $\mathrm{R}$ version 3.1.0. et le package FactoMineR version 1.28 (Lê et al., 2008). Ce package permet l'établissement des analyses multivariées exploratoires ainsi que la classification hiérarchique ascendante.

La typologie des scieurs est dressée en fonction d'un ensemble de variables reprises dans le tableau 1. Ces variables sont de divers ordres: géographique (origine du scieur et localité d'exploitation), écologique (écosystème et espèce), technique (ratio de transformation, possession d'un permis, etc.) et financier (couts, bénéfices financiers, etc.).

L'analyse repose sur une démarche en deux étapes. La première a consisté à structurer et réduire le nombre de variables par l'analyse factorielle des données mixtes (AFDM). L'AFDM permet de faire simultanément un traitement des variables quantitatives et qualitatives en

\footnotetext{
${ }^{1}$ actuelle province Orientale démembrée.
} 


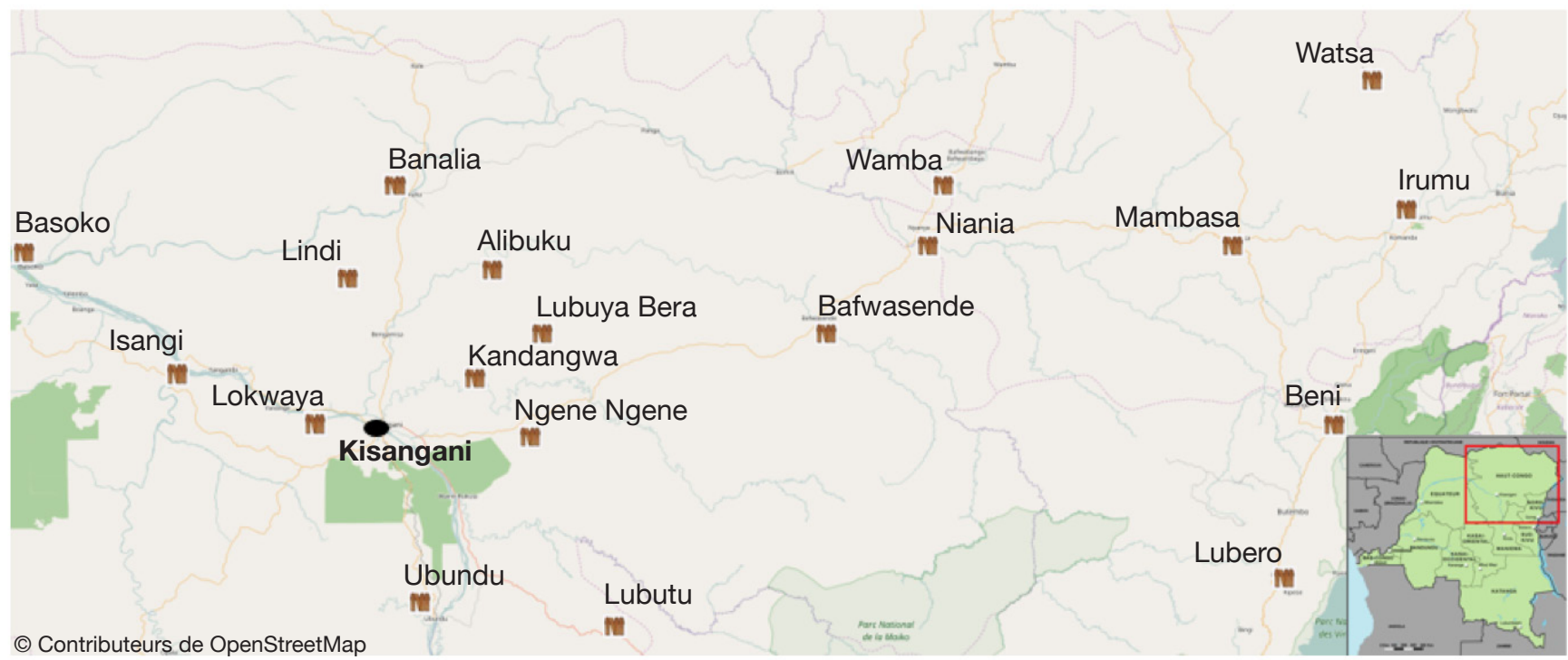

Figure 1. Une partie des sites visités pour les enquêtes en milieu rural dans l'Est de la RDC - Part of the sites visited for rural surveys in the Eastern DRC.

tant que variables actives. L'interprétation des résultats obtenus repose sur l'examen de trois paramètres: l'inertie, la contribution et la liaison entre la variable et l'axe. L'inertie quantifie l'information contenue dans chaque axe. La contribution représente la participation de la variable à la constitution d'un axe. Elle fournit une base pour identifier les variables qui interviennent de façon significative dans la constitution des axes. La liaison entre une variable et un axe traduit la qualité de la représentation (cosinus carré). Pour une variable quantitative, l'intensité de la liaison est évaluée par le niveau de corrélation. Par contre, la liaison entre une variable qualitative et un axe est appréciée par les examens des indicateurs classe/modalité et modalité/ classe ainsi que la valeur-test (v-test).La classe/modalité (cla/mod) indique la part (pourcentage) de tous les individus présentant cette modalité et se retrouvant dans cette classe (cluster, catégorie). Par contre, la modalité/ classe (mod/cla) indique la part (pourcentage) de tous les individus de la classe présentant cette modalité. Une modalité caractérise d'autant mieux une classe que ses deux indicateurs sont grands, simultanément (Lemercier et al., 2010). En effet, la signification de la coordonnée par rapport à zéro est testée à l'aide de la statistique v-test (Morineau, 1984). Dans ce cas, une modalité est considérée comme «significative» dans la constitution d'un axe si sa valeur-test est supérieure à 2 en valeur absolue (au seuil de $5 \%$ ).

La deuxième étape a consisté à établir une classification. Partant des deux premiers axes de l'AFDM, la classification hiérarchique des composants principaux, Hierarchical Classification on Principle Components (HCPC) a été établie. Les valeur-test et probabilités associées à chaque variable et/ou modalité sont utilisées pour déterminer les sous-groupes de scieurs. Chaque variable et/ou modalité est considérée comme «significative» pour déterminer les sousgroupes de scieurs si :

- sa valeur-test est supérieure à 2 en valeur absolue,

- sa probabilité est inférieure à 0,05 ,

- ses indicateurs cla/mod et $\mathrm{mod} / \mathrm{cla}$ sont grands (ou élevés).

Les variables/modalités qui n'ont pas contribué à la constitution des groupes et sous-groupes ont été éliminées automatiquement par le logiciel. Ce qui explique la présence de cellules vides dans les tableaux des résultats.

Les variables « espèces 》 et «écosystèmes » sont traitées comme variables supplémentaires, étant donné qu'elles n'ont pas permis de structurer les données à l'aide de l'AFDM. Ainsi, elles aident à l'interprétation des axes sans en influencer la construction.

\section{RÉSULTATS}

Les valeurs de contributions, coordonnées et cosinus carrés entre les axes 1 et 2 de l'AFDM et chaque variable typologique sont reprises dans le tableau 2. Les deux premiers axes de l'AFDM représentent 59,63\% d'inertie totale. Les variables sont corrélées tantôt 


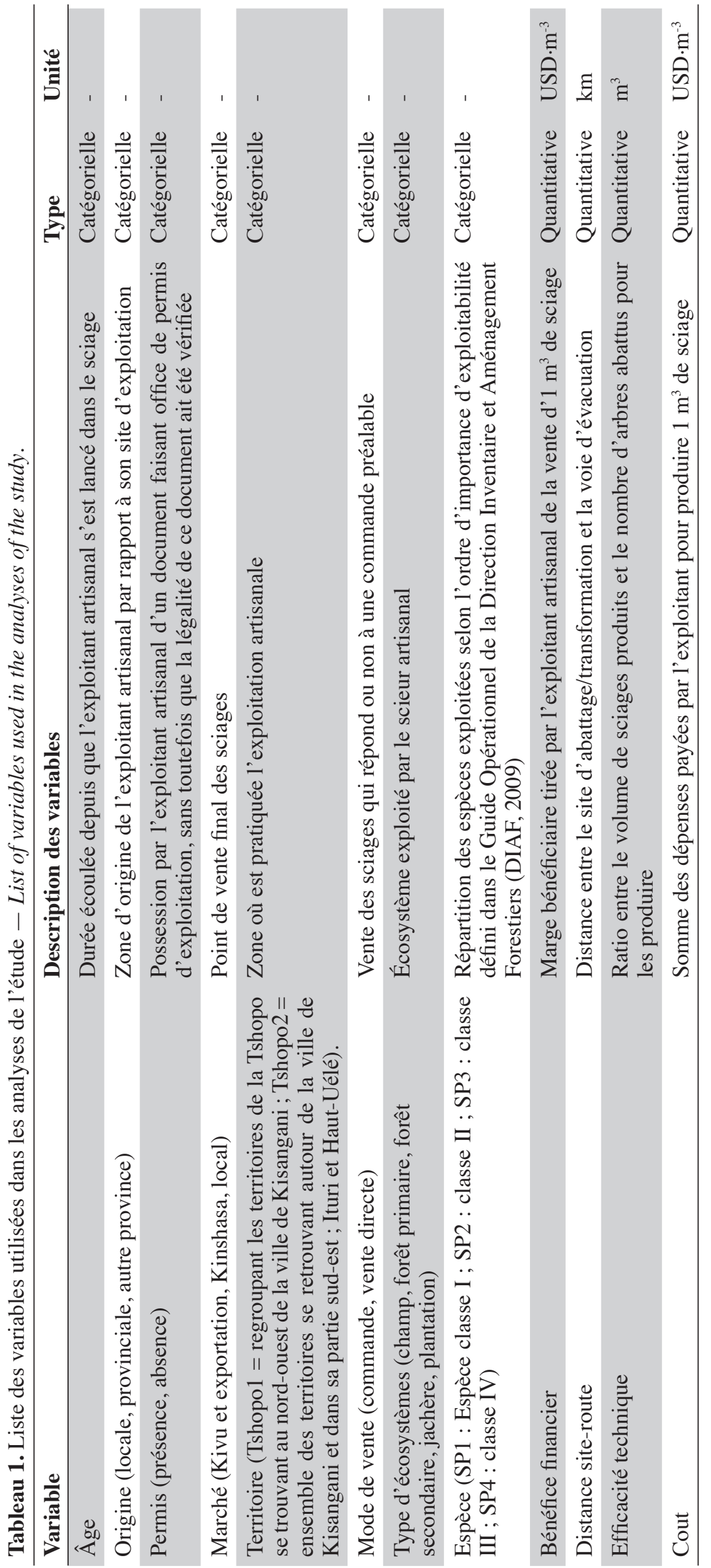

positivement, tantôt négativement avec les axes (Tableau 2). L'axe 1 oppose présence du permis, origine autre province, exportation et marché de Kivu, commande, distance site-route, districts Ituri et Haut-Uélé et dépense (en ordonnée positive) à absence du permis, vente directe, âge, district Tshopo2, origine provinciale, marché de Kinshasa, efficacité technique, consommation locale, origine locale et bénéfice financier (en ordonnée négative) (Tableau 2).

L'axe 2 oppose marché de Kinshasa, origine provinciale, district Tshopo1, âge, dépense et efficacité technique (en ordonnée positive) à origine locale, consommation locale et district Tshopo2 (en ordonnée négative) (Tableau 2).

Partant de l'analyse de classification hiérarchique issue de l'AFDM réalisée sur les deux premiers axes, trois groupes de scieurs ont été identifiés, assortis de sept sous-groupes, comme le montrent la figure 2, les tableaux $3, \mathbf{4 , 5}$ et $\mathbf{6}$.

Le premier groupe (en noir sur la figure 2) rassemble les scieurs d'origine provinciale approvisionnant principalement les marchés de Kinshasa. C'est une ancienne filière puisque la plupart de ses opérateurs ont en moyenne une douzaine d'années d'ancienneté. Ces scieurs recherchent des arbres à des distances inférieures à $3 \mathrm{~km}$ de la route. Ils valorisent davantage les arbres qu'ils abattent en produisant une plus large gamme de produits de toute taille, ce qui se traduit par un ratio élevé de transformation. Leurs niveaux de revenu et de bénéfice financier sont élevés en raison de prix élevés de vente, de la minimisation des couts d'évacuation des sciages de la forêt et du non-payement de permis. Les prix élevés de Kinshasa s'expliquent notamment par un cout important d'accès à la ressource dans les autres sites que la Tshopo approvisionnant Kinshasa en sciage artisanal. D'autre part, Kinshasa constitue le marché dominant pour la vente des sciages artisanaux, principalement en raison de la taille de sa population (Lescuyer et al., 2014).

Ces scieurs sont des vendeurs d'un marché présentant les caractéristiques 
Tableau 2. Valeurs de contributions, coordonnées, cosinus carrés entre les axes 1 et 2 de l'AFDM et chaque variable typologique - Contributions values, coordinates, cosine square between the axes 1 and 2 of the FAMD and each typological variable.

\begin{tabular}{|c|c|c|c|c|c|c|c|c|}
\hline \multirow[t]{2}{*}{ Variables } & \multicolumn{2}{|c|}{ Coordonnées } & \multicolumn{2}{|c|}{ Contributions } & \multicolumn{2}{|c|}{ Cosinus carré } & \multicolumn{2}{|c|}{ Valeur-test } \\
\hline & Axe 1 & Axe 2 & Axe 1 & Axe 2 & Axe 1 & Axe 2 & Axe 1 & Axe 2 \\
\hline \multicolumn{9}{|l|}{ Variables actives } \\
\hline District Haut Uélé & 3,827 & 0,208 & 3,776 & 0,057 & 0,635 & 0,002 & 10,243 & 0,838 \\
\hline District Ituri & 2,436 & 0,013 & 5,032 & 0,001 & 0,795 & 0,000 & 13,361 & 0,110 \\
\hline District Tshopo1 & $-1,894$ & 2,596 & 1,412 & 13,645 & 0,282 & 0,530 & $-6,425$ & 13,264 \\
\hline District Tshopo2 & $-1,560$ & $-0,735$ & 3,599 & 4,106 & 0,760 & 0,169 & $-13,469$ & $-9,554$ \\
\hline Présence de permis & $-1,725$ & $-0,110$ & 5,752 & 0,121 & 0,990 & 0,004 & $-20,399$ & $-1,961$ \\
\hline Absence de permis & 3,192 & 0,204 & 10,645 & 0,223 & 0,990 & 0,004 & 20,399 & 1,961 \\
\hline Origine autre province & 3,192 & 0,204 & 10,645 & 0,223 & 0,990 & 0,004 & 20,399 & 1,961 \\
\hline Origine locale & $-1,484$ & $-1,646$ & 2,510 & 15,887 & 0,436 & 0,537 & $-10,158$ & $-16,970$ \\
\hline Origine provinciale & $-2,070$ & 2,095 & 3,403 & 17,930 & 0,486 & 0,498 & $-10,850$ & 16,539 \\
\hline Consommation locale & $-1,492$ & $-1,570$ & 2,659 & 15,146 & 0,465 & 0,515 & $-10,613$ & $-16,820$ \\
\hline Marché de Kinshasa & $-2,100$ & 2,248 & 3,262 & 19,220 & 0,460 & 0,526 & $-10,494$ & 16,915 \\
\hline Kivu et exportation & 3,192 & 0,204 & 10,645 & 0,223 & 0,990 & 0,004 & 20,399 & 1,961 \\
\hline Commande & 3,192 & 0,204 & 10,645 & 0,223 & 0,990 & 0,004 & 20,399 & 1,961 \\
\hline Vente directe & $-1,725$ & $-0,110$ & 5,752 & 0,121 & 0,990 & 0,004 & $-20,399$ & $-1,961$ \\
\hline Bénéfice financier & $-0,337$ & 0,140 & 1,956 & 0,764 & 0,113 & 0,020 & - & - \\
\hline Dépense & 0,385 & 0,300 & 2,556 & 3,520 & 0,148 & 0,090 & - & - \\
\hline Distance site-route & 0,603 & 0,090 & 6,273 & 0,316 & 0,363 & 0,008 & - & - \\
\hline Efficacité technique & $-0,547$ & 0,166 & 5,157 & 1,073 & 0,299 & 0,027 & - & - \\
\hline Âge & $-0,500$ & 0,429 & 4,319 & 7,202 & 0,250 & 0,184 & - & - \\
\hline \multicolumn{9}{|l|}{ Variables supplémentaires } \\
\hline Espèce & 0,222 & 0,163 & - & - & 0,016 & 0,009 & - & - \\
\hline Écosystème & 0,338 & 0,669 & - & - & 0,029 & 0,112 & - & - \\
\hline
\end{tabular}
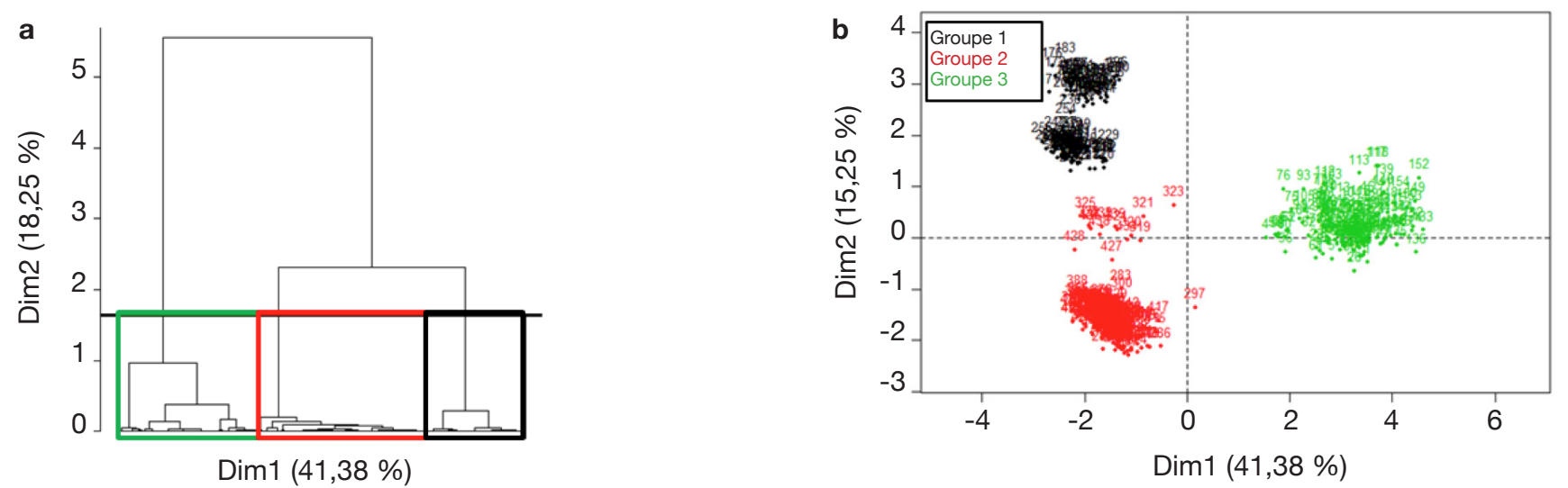

Figure 2. Visualisation des groupes de scieurs à partir de la classification hiérarchique des composantes principales effectuée à l'aide de deux axes de l'analyse factorielles des données mixtes - Visualisation of groups loggers from the hierarchical classification of principal components made using the first two axes of the factorial analysis of mixed data.

d'un oligopole, c'est-à-dire un petit nombre de vendeurs face à une multitude d'acheteurs (Figure 2, Tableau 3). Ce premier groupe se subdivise en deux sous-groupes.

Le premier sous-groupe rassemble les scieurs situés dans les districts de la Tshopo2. Ces scieurs dégagent un bénéfice financier élevé qui tient à :
- une gamme d'espèces exploitées et rencontrées en forêt secondaire à moins de $3 \mathrm{~km}$;

- des couts d'exploitation réduits suite à la minimisation de la distance d'évacuation (Tableau 4).

Le deuxième sous-groupe est constitué des scieurs opérant dans le district de la Tshopo1 et recherchant une 
Typologie des scieurs artisanaux en province Orientale

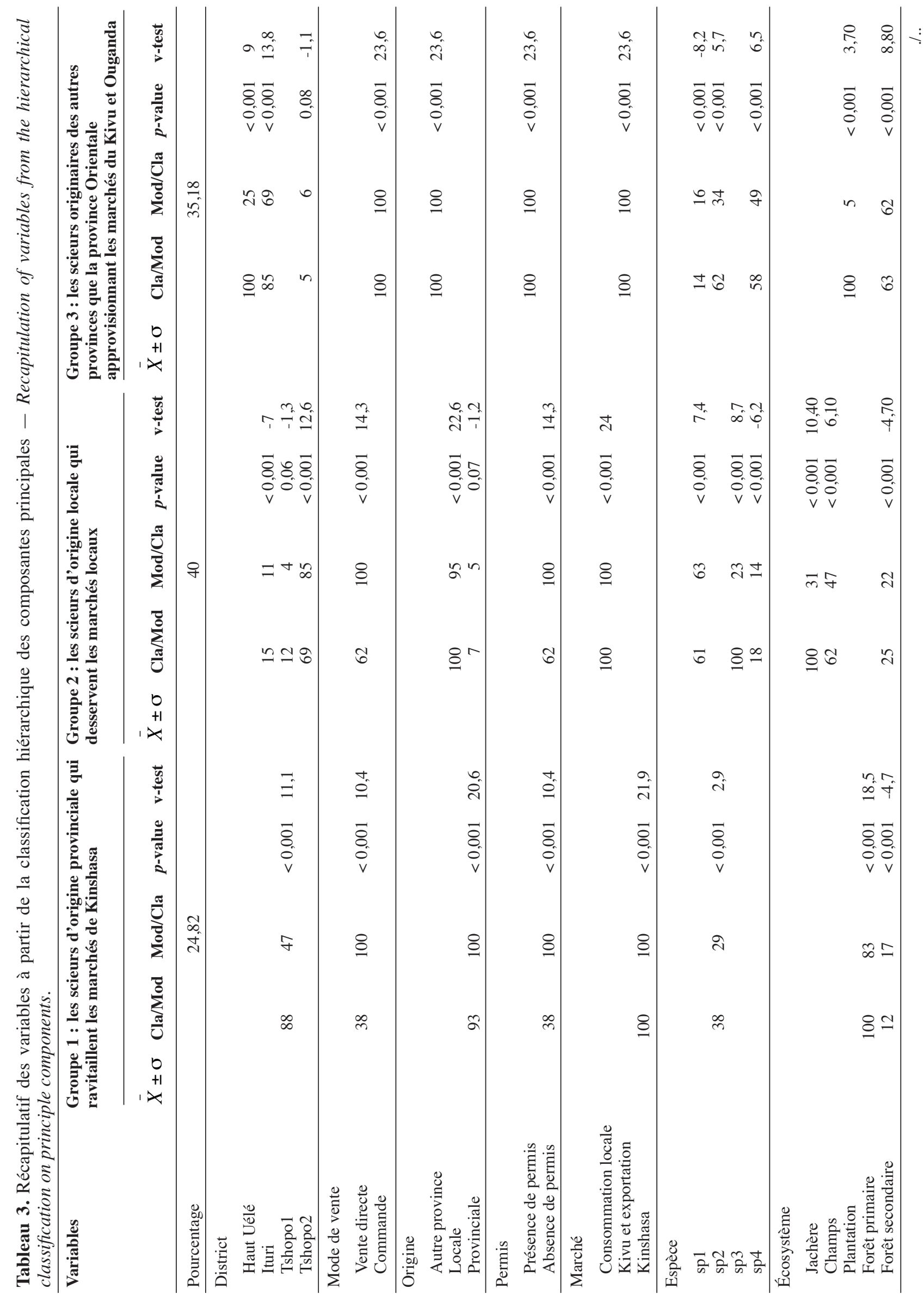


poignée d'essences de bois nobles : Entandrophragma utile (Dawe \& Sprague) Sprague, Khaya anthotheca (Welw.) C.DC., Pericopsis elata (Harms) Meeuwen, Pterocarpus soyauxii Taub., Milicia excelsa (Welw.) C.C.Berg. Il réalise globalement un bénéfice financier positif par la minimisation de la distance de la voie d'évacuation et de sa spécialisation dans l'exploitation d'une poignée d'essences de bois nobles (Tableau 4).

Le deuxième groupe (en rouge sur la figure 2) réunit les scieurs d'origine locale qui approvisionnent les marchés locaux. Leurs produits sont destinés à la vente directe (auprès de constructeurs, menuisiers, etc.). Opérant dans l'informel, ces acteurs exploitent une large gamme d'espèces ligneuses dont les arbres proviennent, dans la plupart des cas, des champs, jachères et forêts secondaires situés à moins de $2 \mathrm{~km}$ de la voie d'évacuation. Pour répondre à une demande peu exigeante en termes de qualité, ces scieurs obtiennent un excellent taux de transformation et un bénéfice financier élevé (Figure 2, Tableau 3). Ce deuxième groupe comprend également deux sous-groupes.

Le premier sous-groupe rassemble les scieurs exerçant leurs activités dans les districts de la Tshopo2 et d'Ituri. Ceux-ci recherchent prioritairement les essences de bois nobles localisées dans les champs, jachères et forêts exploitées, distants de $2 \mathrm{~km}$ de la voie d'évacuation (Tableau 5).

Le deuxième sous-groupe réunit des scieurs opérant dans le district de la Tshopo1. Globalement, ces opérateurs exploitent les arbres situés en zones agricoles et dégagent un bénéfice financier élevé après avoir engagé d'importantes dépenses (Tableau 5).

Le troisième groupe (en vert sur la figure 2) est constitué des scieurs provenant des autres provinces que la province Orientale. Les produits de ces scieurs sont destinés aux marchés des villes du Kivu et à l'exportation vers l'Ouganda. Nombre de ces scieurs sont employés par des commanditaires basés au Kivu, en Ouganda et au Kenya. Ces derniers leur assurent l'achat des sciages tout en finançant fréquemment l'octroi d'un permis d'exploitation artisanale, l'exploitant local étant rarement en mesure de dépenser plus de 3000 USD pour démarrer son activité. En plus du capital, ces investisseurs étrangers fournissent aux scieurs des tronçonneuses. Ces commanditaires facilitent l'importation des machines, du carburant et autres marchandises de leurs voisins orientaux (Forests Monitor, 2007; Umunay et al., 2009). C'est une jeune filière puisque ces opérateurs sont caractérisés par une moyenne d'ancienneté de 6 ans. Ces scieurs réalisent globalement une perte liée au cout élevé d'exploitation (notamment les transports sur de grandes distances) et le bas niveau des prix de vente fixés par leurs patrons du Kivu ou d'Ouganda. Polepole (2008) révèle l'existence d'un grand nombre de taxes (jusqu'à 34 en Ituri) grevant l'exploitation artisanale du bois. La 
Typologie des scieurs artisanaux en province Orientale

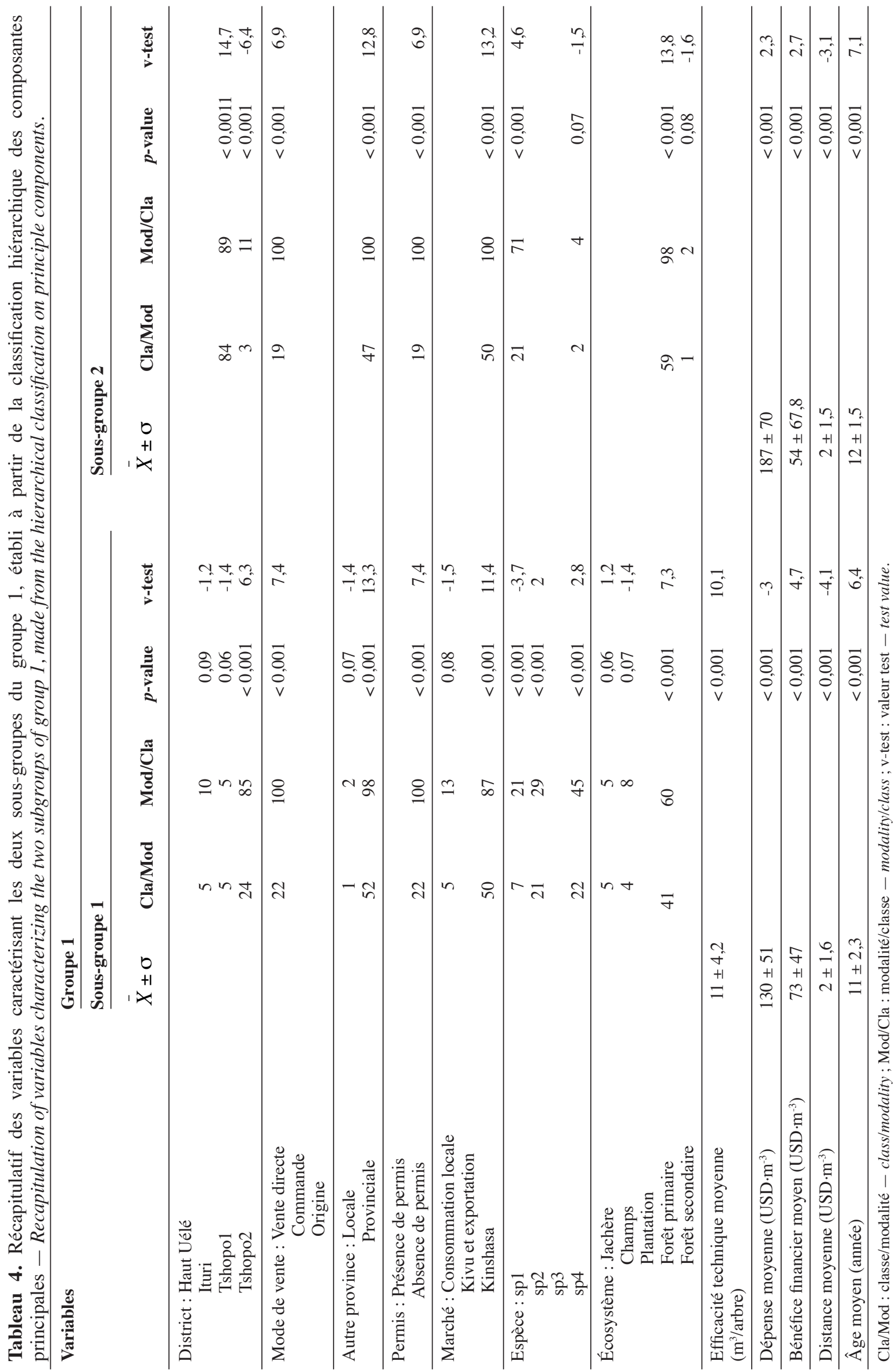


Tshimpanga O., Lescuyer G., Vleminckx J. et al.

क्ष
के

ฮิ

릉

䨌

인

.ำ

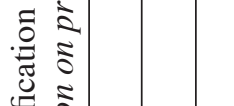

章

$\approx$

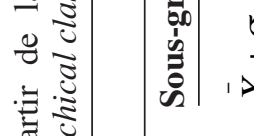

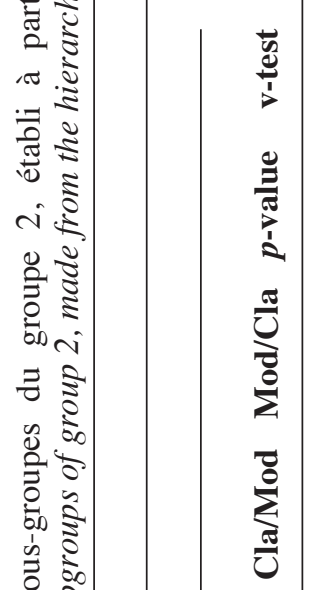

$\times$

छे

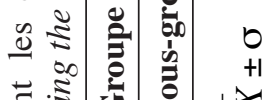

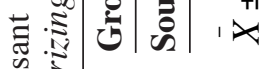

:

范

ฮิ

$\frac{0}{0} \frac{a}{0} \cdot \frac{5}{3}$

灵

के

0 .

表

言

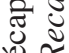

\&

व.।

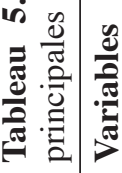

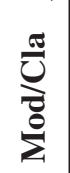

$\sum_{\frac{\pi}{0}}^{\bar{g}}$

๕ั้

o

$b$
+1
$x$
ले $\mathrm{N}$

$\overline{8}$
0
$v$

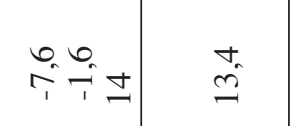

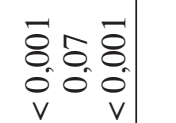

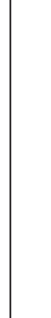

$\infty$

$\sqrt{2}$

$\circ \cdots$

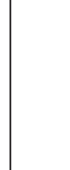

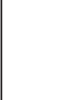

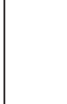

. $\overrightarrow{8}$
$\dot{0}$
$v$

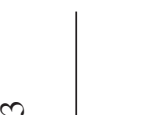

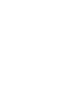

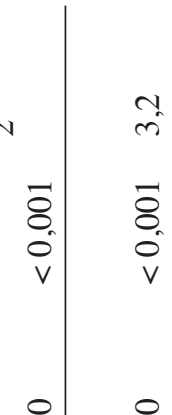

8 \&

तेन

$\begin{array}{ll}\overline{8} & 8 \\ 0 & 0 \\ v & 0\end{array}$

$\begin{array}{ll}\overrightarrow{8} & \overline{8} \\ 0 & 0 \\ 0 & v\end{array}$

i

8

a-

8

ㄴ m.

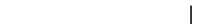

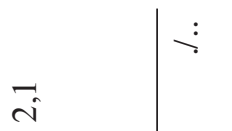

ন

$\overrightarrow{8}$
$\dot{0}$
$v$

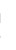

के तुथ के के

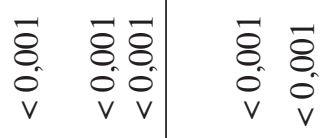

ชูก

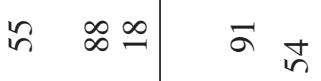


plupart de ces taxes sont déterminées par le volume produit ou transporté. Elles sont prélevées à l'endroit du chargement des bois sciés dans les camions et le long de la route. La majorité de la perception de ces taxes ne fait pas l'objet d'une quittance et ne constitue donc pas une recette pour le Trésor Public (Forests Monitor, 2007).

Les exploitants de ce troisième groupe récoltent un spectre assez large d'espèces recherchées jusqu'à $5 \mathrm{~km}$ en forêt déjà exploitée. Leur ratio de transformation est faible car ils produisent du sciage de grosse taille, qui sera profilé de nouveau une fois parvenu aux marchés urbains. Ce sciage est accaparé par un petit nombre d'intermédiaires qui l'achètent à un bas prix auprès des scieurs, formant ainsi un oligopsone. Ces prix de vente représentent environ la moitié du prix de produits rendus aux frontières et moins du tiers du prix sur les marchés étrangers de Kampala ou de Nairobi (Lescuyer, 2010). Il s'ensuit une spirale d'endettement de l'exploitant qui n'est plus en mesure de discuter avec son «patron » le prix du bois qu'il lui fait parvenir pour rembourser (toujours partiellement) ses dettes. Depuis 2006, le marché du bois, jadis situé géographiquement en RDC, a été transféré en Ouganda et y est resté depuis lors. Les exploitants trouvent que ce déplacement du marché du bois a entrainé une augmentation de la fraude, du chômage et des vols. Cette situation qui perdure est observée non seulement pour le bois mais aussi pour divers produits alimentaires et pour les minerais (Forests Monitor, 2007). Un autre effet de cette relation asymétrique patron-client demeure la sous-estimation systématique des volumes vendus par les exploitants artisanaux. Sous prétexte que les pièces livrées devront être profilées de nouveau et parfois redimensionnées, les acheteurs-patrons appliquent des standards fictifs de cubage qui minimisent le volume réel (de $9 \%$ en moyenne dans la présente enquête, mais certains cas peuvent aller jusqu'à $60 \%$ [Lescuyer, 2010]). La combinaison d'un bas prix d'achat au $\mathrm{m}^{3}$ et d'une sousestimation du volume vise à maintenir les exploitants artisanaux sous la coupe des acheteurs-entrepreneurs du Kivu et des pays voisins (Figure 2, tableau 3). Ce troisième groupe est fragmenté en trois sous-groupes.

Le sous-groupe 1 est constitué des scieurs localisés dans les districts d'Ituri et de Tshopo2, abattant des arbres localisés principalement dans des forêts dégradées et des plantations (Tableau 6).

Le sous-groupe 2 réunit des scieurs exerçant dans le district d'Ituri. Ceux-ci recherchent des espèces de grande valeur commerciale qu'ils trouvent, grâce à leurs relations personnelles, en forêt secondaire et dans les champs, et à $4 \mathrm{~km}$ de la voie d'évacuation. Avec un cout d'exploitation élevé, ces scieurs valorisent davantage les arbres abattus en produisant une plus large gamme de produits de toute taille, ce qui explique leur bon ratio de transformation. C'est aussi une jeune 
Tshimpanga O., Lescuyer G., Vleminckx J. et al.

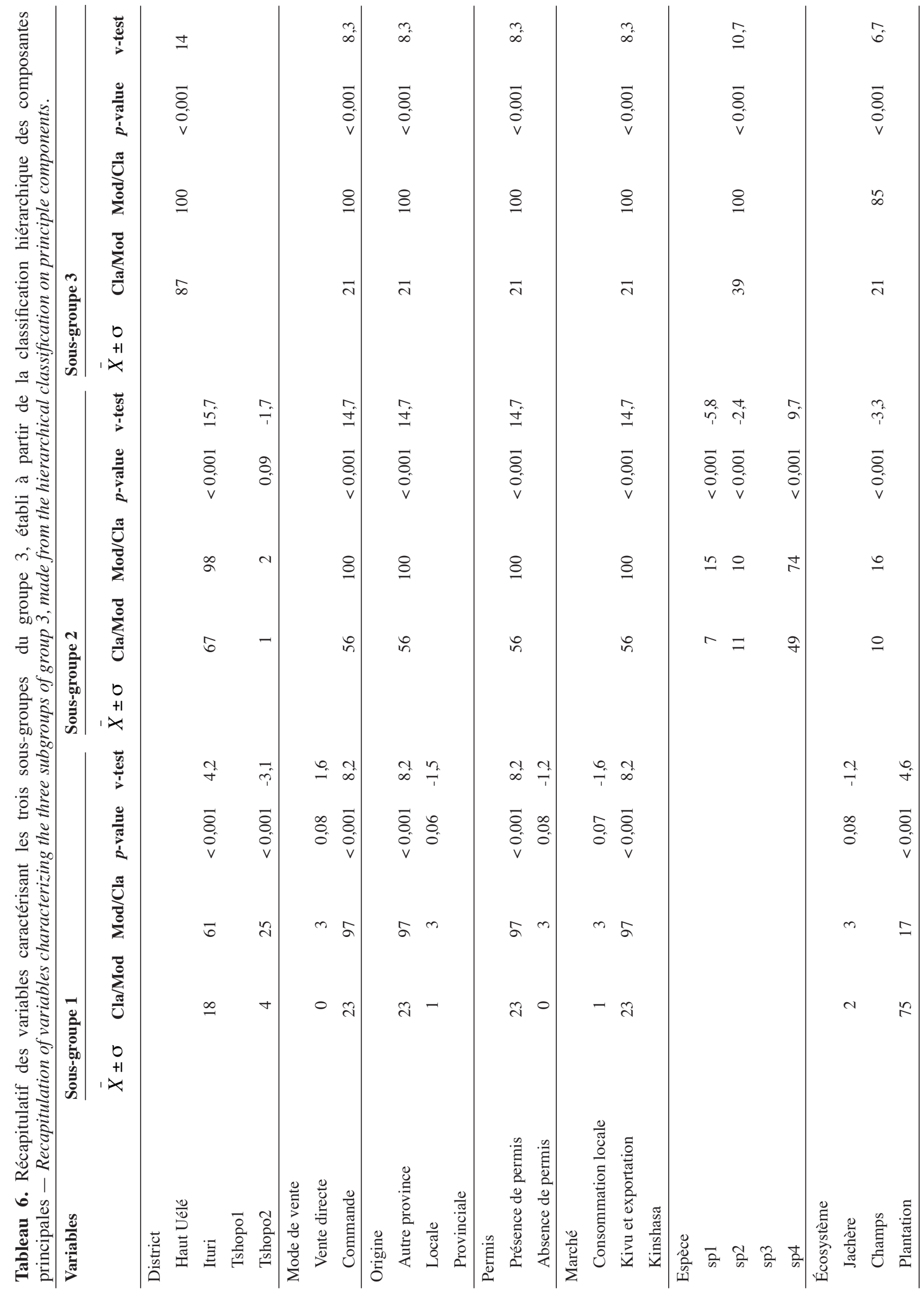




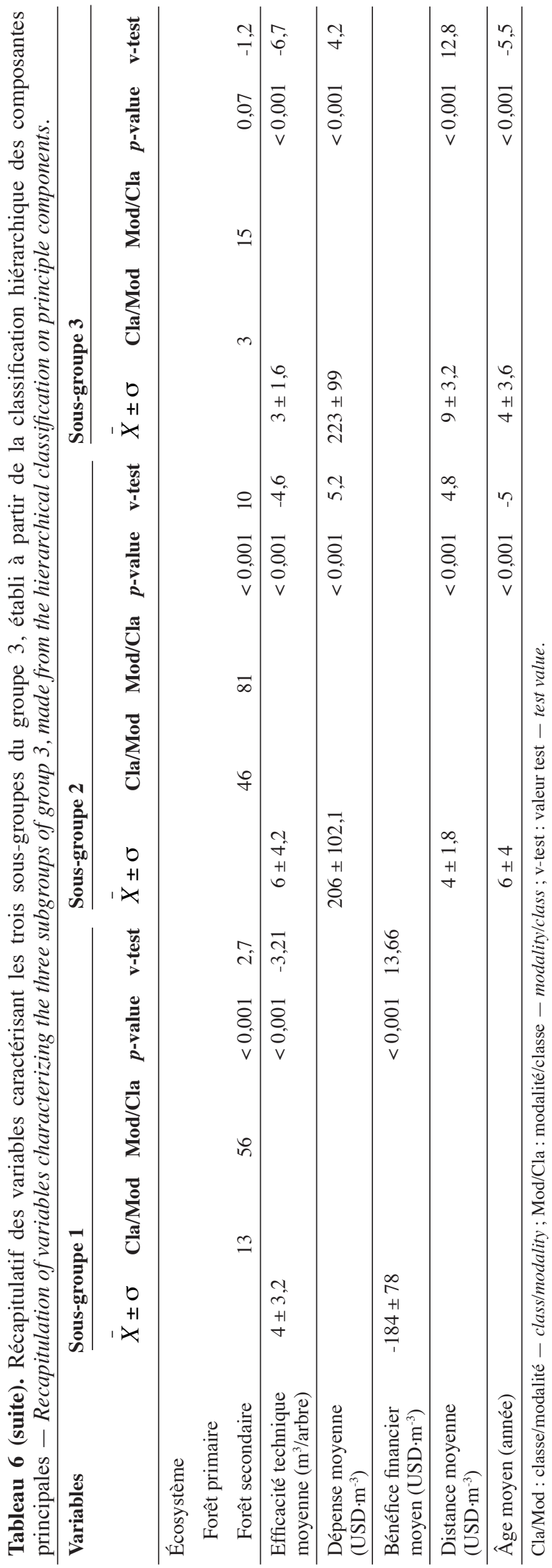

filière avec une moyenne de 6 années d'ancienneté (Tableau 6).

Le sous-groupe 3 rassemble des scieurs opérant dans le district de Haut-Uélé. Ces opérateurs supportent un cout d'exploitation le plus élevé de tous : ils exploitent un petit nombre d'espèces ligneuses dont les arbres proviennent, dans la plupart des cas, de champs situés le plus souvent à $9 \mathrm{~km}$ de la voie d'évacuation et ils obtiennent généralement l'efficacité technique la plus faible (Tableau 6).

\section{DISCUSSION}

L'examen de la filière des scieurs artisanaux actifs en province Orientale montre combien leur typologie est hétérogène. Plutôt qu'un traitement homogène du secteur du sciage informel, la politique publique gagnerait en efficacité et en pertinence si elle était élaborée sur la base d'une meilleure compréhension de la variété de configurations dégagées à l'issue de la présente analyse.

Partant de la diversité de ces scieurs, cinq volets ont été considérés dans l'élaboration et la mise en œuvre d'une politique forestière en RDC.

Tout d'abord, la formalisation de l'exploitation artisanale des forêts est un enjeu majeur pour la RDC. En province Orientale, seuls les scieurs de groupe 3 détiennent majoritairement des documents faisant office de permis, leur donnant ainsi la capacité d'expédier leurs produits vers les marchés du Kivu (groupe 3) ou d'accéder à des zones d'exploitation dont ils ne sont pas originaires (groupe 3), tandis que les autres opèrent sans permis et exploitent les arbres se trouvant dans les zones d'exploitation dont ils sont originaires (groupes 1 et 2). Ces scieurs présentent des profits négatifs liés en partie à l'accès onéreux des permis de coupe (groupe 3).

Les exploitants artisanaux supportent des couts fixes pour accéder de manière officielle à la ressource. Solliciter un permis d'exploitation artisanale implique des dépenses estimées à 3000 USD (Lescuyer, 2010). En outre, l'exploitant doit réaliser un cahier des charges détaillant ce qu'il doit fournir en nature à la population. La valeur totale des dépenses consenties dans le cadre du cahier des charges s'établit en moyenne à 1960 USD (Lescuyer et al., 2014).

En province Orientale, l'accès à la légalité pour les scieurs artisanaux bute sur une difficulté relative aux groupes cibles qui devront supporter l'effort de la légalisation de l'activité. Il s'agit des scieurs d'origine locale (groupe 2) ou de ceux approvisionnant les demandes locales (groupe 2). La lutte contre l'exploitation artisanale informelle touche donc un public qui bénéficie d'une proximité géographique et politique avec les autorités provinciales. Dans ces 
circonstances, il est peu probable que le Ministère provincial en charge des forêts puisse lutter efficacement contre le sciage informel sans un soutien de la part du ministère national des forêts ou d'une société civile en mesure d'agir au-delà de la collusion d'intérêts locaux.

Deuxièmement, l'exploitation artisanale doit également contribuer à l'augmentation des revenus des populations locales (Benneker et al., 2012; Tiayon et al., 2012). Eu égard à leur niveau élevé de profit, les scieurs des groupes 1 et 2 pourraient attribuer une part plus importante de leurs recettes aux communautés. Cela supposerait de clarifier et de réviser la règlementation encadrant l'exploitation des arbres dans les zones agricoles, notamment la possibilité pour les propriétaires traditionnels de faire un usage commercial des arbres qui sont situés dans les espaces coutumiers sur lesquels ils exercent des maitrises foncières et dans le domaine rural.

Troisièmement, l'augmentation des recettes fiscales est également un des résultats attendus de la formalisation de l'exploitation artisanale (Benneker et al., 2012; Tiayon et al., 2012). Elle sera d'autant plus acceptable qu'elle porte sur les groupes de scieurs dégageant un profit élevé (groupes 1 et 2). $\mathrm{Au}$ demeurant, les sciages transitent (groupe 1) ou sont vendus (groupe 2) à Kisangani, ce qui facilite la mise en place d'une taxation localisée dont le recouvrement est relativement simple. Une telle taxation ne pourrait intervenir qu'après la réduction du cout actuel du permis d'exploitation.

Quatrièmement, la valorisation accrue des arbres est un autre objectif de la politique forestière congolaise (Sakata, 2007). Si l'efficacité technique du sciage artisanal est globalement élevée, les scieurs approvisionnant les marchés du Kivu (groupe 3) seront nettement moins efficients que les autres groupes de scieurs car ils sont contraints par leurs patrons de ne livrer que des pièces de volume important. Un des objectifs spécifiques de la politique forestière provinciale devrait être alors de faciliter l'accès pour ces scieurs aux marchés du Kivu et de l'Ouganda en leur permettant de rompre leur dépendance vis-à-vis des patrons extérieurs. Cela suppose par exemple d'appuyer la constitution d'associations indépendantes de scieurs et de leur ouvrir des lignes de crédits pour leur permettre de couvrir par eux-mêmes les frais d'accès formels et informels à ces marchés lointains (Lescuyer, 2010).

Enfin, l'exploitation artisanale doit permettre la régénération des ressources ligneuses exploitées. À ce jour, les scieurs artisanaux font preuve d'une remarquable efficacité technique, qui s'explique notamment par la grande taille des arbres abattus. La focalisation du sciage artisanal sur les gros arbres indique que ces arbres sont encore disponibles dans les zones étudiées et que les plus petits ne font pas encore l'objet d'une exploitation à grande échelle. Un des enjeux en termes de durabilité de la ressource est de diversifier les espèces exploitées, notamment pour le groupe 2 de scieurs qui se focalise aujourd'hui sur un petit nombre d'espèces à destination des marchés locaux. C'est à ces demandes urbaines spécifiques que doivent s'adresser des campagnes de sensibilisation et de démonstration, si on veut élargir la palette des espèces abattues et faire baisser la pression sur les essences à forte valeur commerciale. Il faut également imposer certaines pratiques sylvicoles à toutes les catégories de scieurs, notamment le respect strict des Diamètres Minimum d'Exploitation (DME) des essences protégées, ainsi qu'un reboisement. Toutefois, des études approfondies sur les impacts environnementaux occasionnés par le sciage artisanal à petite échelle sont nécessaires afin de confirmer que cette pratique dévalorise la valeur de massifs forestiers concernés et contribue à la dégradation des forêts.

\section{CONCLUSIONS}

Dans la province Orientale, depuis une quinzaine d'années, on constate l'essor d'un secteur de sciage artisanal essentiellement informel, approvisionnant les marchés provinciaux et certains marchés étrangers. Une analyse statistique des pratiques des scieurs artisanaux permet de les classer en trois types d'opérateurs :

- les scieurs d'origine provinciale qui ravitaillent les marchés de Kinshasa ;

- les scieurs d'origine locale qui desservent les marchés locaux ;

- les scieurs originaires des autres provinces que la province Orientale qui approvisionnent les marchés du Kivu et de l'Ouganda.

La RDC négocie actuellement avec l'Union européenne pour établir à terme un Accord de Partenariat Volontaire visant à assurer la légalité et la durabilité de l'exploitation forestière. Le secteur du sciage artisanal est au cour de ces discussions et devra faire l'objet d'une stratégie spécifique à l'échelle nationale, comme aux échelles des provinces forestières. En province Orientale, il n'existe pas aujourd'hui de politique spécifique pour formaliser l'exploitation artisanale du bois. Réfléchir à la mise en ouvre de la politique publique sur la base d'une bonne compréhension de la typologie des scieurs artisanaux permettrait d'améliorer la pertinence et l'efficacité des instruments de légalisation et de renforcement de l'exploitation artisanale du bois en RDC. 


\section{Remerciements}

La présente publication n'aurait pu se faire sans l'appui des projets UE « Pro-Formal » (EuropeAid/ENV/2010-242904/ TPS ) et « REFORCO » (FED/2009/219-879).

\section{Bibliographie}

Bayol N. et al., 2012. La gestion des forêts et la filière bois en Afrique Centrale. In : de Wasseige C. et al., eds. Les forêts du bassin du Congo. État des forêts 2010. Luxembourg : Office des Publications de l'Union européenne, 43-46.

Belesi H., Enyuka G. \& Kiyulu J., 2013. Filière bois artisanal dans la province du Bas-Congo. Interactions des acteurs et impact environnemental dans les territoires de Mbanza Ngungu, Songololo, Seke Banza, Lukula, Tshela et Moanda. Rapport IUCN-AFD, Kinshasa, RDC.

Benneker C. et al., 2012. Le bois à l'ordre du jour. Exploitation artisanale de bois d'œuvre en RD Congo : secteur porteur d'espoir pour le développement des petites et moyennes entreprises. Wageningen, Pays-Bas : Tropenbos.

Debroux L. et al., 2007. Forests in post-conflict Democratic Republic of Congo. Bogor, Indonesia: Center for International Forestry Research, the World Bank.

DIAF, 2009. Guide opérationnel, listes des essences forestières de la République Démocratique du Congo. Kinshasa, RDC : Direction Inventaire et Aménagement Forestiers.

Djiré A., 2003. Étude sur le secteur informel du bois d'œuvre en RDC. Rapport d'appui à la revue économique du secteur forestier en RDC. Montpellier, France : CIRAD.

Forests Monitor, 2007. Commerce du bois et réduction de la pauvreté. Région des Grands Lacs. Rapport. Londres : Forests Monitor.

Global Witness, 2012. The art of logging industrially in the Congo: How loggers are abusing artisanal permits to exploit the DRC's forests. London: Global Witness.

Lê S., Josse J. \& Husson F., 2008. FactoMineR: an R package for multivariate analysis. J. Stat. Software, 25(1), 1-18.

Lemercier C., Milani P. \& Sofio S., 2010. Tutoriel FactoMineR pour l'analyse des correspondances multiples avec une petite annexe sur la classification automatique, http://www.quanti.ihmc.ens.fr/IMG/pdf/ Tutoriel_FactomineR_ACM, (20/02/2014).
Lescuyer G., 2010. Analyse économique de l'exploitation forestière artisanale dans la Province Orientale de la République Démocratique du Congo: diagnostic succinct. Londres : Forests Monitor.

Lescuyer G. et al., 2014. The domestic market for smallscale chainsaw milling in the Democratic Republic of Congo: present situation, opportunities and challenges. Occasional Paper 112. Bogor, Indonesia: Center for International Forestry Research, http:/www.cifor. org/publications/pdf_files/OccPapers/OP-112.pdf (18/12/2015).

Morineau A., 1984. Note sur la caractérisation statistique d'une classe par les valeurs tests. Bull. Techn. CESIA, 2(1), 20-27.

Nkoy E., 2007. Exploitation du bois, paradoxe de la pauvreté et conflits dans le Territoire de Mambasa (Ituri, Nord-Est de la RDC). Rapport pour IKV-Pax Christi Pays-Bas. Kinshasa, RDC.

Polepole P., 2008. Analyse et commentaires des politiques et textes en matière de l'exploitation forestière artisanale. Rapport UICN-USAID-WCS. Kinshasa, RDC.

Sakata G., 2007. Le code forestier et le code minier de la RDC : rôle des acteurs et impact socio-économique. Essai comparatif. Tervuren, Belgique.

Tiayon F. \& Molnar A., 2012. Perspectives comparatives de l'exploitation artisanale du bois en RD Congo :évolution des politiques et des pratiques. In : Benneker C. et al., eds. Le bois à l'ordre du jour. Exploitation artisanale de bois d'œuvre en RD Congo : secteur porteur d'espoir pour le développement des petites et moyennes entreprises. Wageningen, Pays-Bas : Tropenbos, 133-135.

Umunay P. \& Makana J.R., 2009. Étude sur le commerce transfrontalier de bois dans le Nord-Est de la République Démocratique du Congo. Rapport UICN, 23.

Van Acker F., 2013. Analyse critique de la gestion de l'écosystème forestier en RDC. In: Maryss E.S. \& Omasambo J., eds. Conjonctures congolaises 2012. Politiques, secteur minier et gestion des ressources naturelles en RD Congo. (coll. «Cahiers africains», $n^{\circ}$ 82). Tervuren, Belgique : Musée Royal de l'Afrique Centrale ; Paris : Éditions L’Harmattan, 179-209. 